\title{
Effect of Psyllium Husk, Bran, and Raw Wheat Germ Addition on the Rheological Characteristics of Arabic (Pita) Bread Dough
}

\author{
Ahmad Aldughpassi $\mathbb{D}^{1},{ }^{1}$ Tasleem Zafar $\mathbb{D}^{1},{ }^{1}$ Jiwan S. Sidhu $\mathbb{D}^{1,2}$ Fatima Al-Hassawi ${ }^{1},^{1}$ \\ Mohammad Mirza Abdullah $\left(\mathbb{D},{ }^{1}\right.$ and Amani Al-Othman ${ }^{3}{ }^{3}$ \\ ${ }^{1}$ Department of Food Science \& Nutrition, College of Life Sciences, Kuwait University, P.O. Box. 5969, Safat, 13060, Kuwait \\ ${ }^{2}$ Food Science \& Nutrition Program, P.O. Box. 24885, Safat, 13109, Kuwait \\ ${ }^{3}$ Information and Communications Technology Dept, Kuwait Institute for Scientific Research, P.O. Box. 24885, Safat, 13109, Kuwait
}

Correspondence should be addressed to Jiwan S. Sidhu; jiwan.sidhu@yahoo.com

Received 3 September 2020; Revised 30 November 2020; Accepted 8 December 2020; Published 30 December 2020

Academic Editor: Giorgia Spigno

Copyright (c) 2020 Ahmad Aldughpassi et al. This is an open access article distributed under the Creative Commons Attribution License, which permits unrestricted use, distribution, and reproduction in any medium, provided the original work is properly cited.

\begin{abstract}
Arabic bread (khubuz) made from white flour is the staple food in the Arabic countries but has now become popular all over the world. A different approach of producing high fiber bread with improved quality can be produced using white flour with added mill fractions, but the addition of mill fractions has been shown to adversely affect the dough characteristics. Therefore, the effect of adding mill fractions on the rheological characteristics of dough was investigated using Brabender Farinograph and Extensograph with the major objective of eliminating their deleterious effects on dough quality, mainly by using psyllium husk, and also reported as an excellent source of soluble dietary fiber. Addition of fine bran, coarse bran, and raw wheat germ decreased the extensibility and resistance to extension and area under curve, lower dough stability, but enhanced water absorption and peak time. Addition of psyllium husk, though reduced the extensibility, but did not affect the area under the curve adversely, thus overcame some of the negative effects on rheological characteristics of the white flour dough. It was concluded that the use of psyllium husk will evidently help the bakers to produce nutritious and acceptable quality Arabic bread.
\end{abstract}

\section{Introduction}

Most of the nations have now established grains as the base of healthy eating pattern and are recommending multiple servings of whole grains [1]. Moreover, whole grains are rich source of many antioxidants which protect us against some of the chronic diseases, including cardiovascular diseases, diabetes, and many types of cancer [2]. Whole wheat flour and whole wheat bread are reported to lower cholesterol in rats [3], apart from providing a wide range of nutrients and biologically active compounds such as dietary fiber, B and E vitamins, selenium, zinc, copper, magnesium, and phenolic compounds, which work synergistically to reduce the incidence of various diseases [4]. Bile binding by wheat bran may contribute to cancer prevention and other healthful properties [5].
The Kuwaiti population mainly consumes white Arabic bread (khubuz), white toast bread, and highly polished rice; this evidently results in lower intake of dietary fiber. The incidence of constipation and its related diseases like diverticular, appendicitis, piles, hemorrhoids, and anal fissures is significantly high among children, adults, and the elderly in the Kuwaiti population. This necessitates the need for commonly consumed food products such as Arabic bread enriched with both the soluble and insoluble dietary fiber sources. According to the latest figures available [6], a total of 24,300 inpatients (diabetics, and cardiac, hypertension and cancer cases) had to be served with daily meals having high fiber contents in the various clinics of the country. The catering companies would have to produce nearly 16,000 loaves of high fiber Arabic bread daily to meet these requirements. In addition, many thousands of outpatients 
visiting weight clinics would like to consume such high-fiber baked products.

The consumption of toast bread is steadily increasing at about $10 \%$ per annum, whereas Arabic bread consumption has almost stagnated. More than $90 \%$ of the bread being consumed in Kuwait is made from white flour, which is depleted of natural dietary fiber. The total dietary fiber content of whole wheat flour is $10.2 \%$ compared with $2.5 \%$ for white flour. On the other hand, the values for total dietary fiber in wheat bran range between 40 and $44 \%$, thus making it an ideal natural supplement for producing high-fiber baked products [7]. The Kuwaiti consumers prefer white flour Arabic bread over that of whole wheat flour bread, mainly because of its lighter color and superior eating quality. Use of psyllium is expected not to affect the crust color of these baked products adversely, thus retaining their consumer acceptability.

The satiety value of whole meal bread has been reported to be significantly higher than white bread [8]. Thus, one would eat more white bread leading to higher calorie intake, as white bread has a greater energy density $(2.3 \mathrm{cal} . / \mathrm{g})$ than whole meal bread $(2.16 \mathrm{cal} . / \mathrm{g})$. In general, subjects consuming whole meal or high-fiber breads feel fuller than those consuming white bread, as the former has higher satiety value. Wheat bran and germ, because of their flavor, and a good amount of proteins of high biological value, have also been reported to be a rich source of B-complex vitamins and minerals [9]. The dough rheological characteristics are good predictors of the quality of the finished products (Arabic bread), but the addition of coarse bran, fine bran, and raw wheat germ has been reported to adversely affect the rheological performance of wheat dough [7]. The major objective of this investigation was to optimize the use of psyllium husk to eliminate the adverse effects of addition of mill fractions on the rheological characteristics of wheat flour dough required for Arabic bread making.

\section{Materials and Methods}

2.1. Raw Materials. Whole wheat flour (WWF), white flour (WF), coarse bran (CB), fine bran (FB), and wheat germ (WG) samples were obtained from the Kuwait Flour Mills \& Bakeries Co., Shuwaikh. Psyllium husk was procured from India through a local importer. These samples were analyzed for moisture (method 44-19), crude protein (method 46-12), crude fat (method 30-25), and ash (method 08-01) contents, according to standard AACC methods [10]. Coarse bran fraction comes from the outer layers of wheat grain and is obtained from the break roll section, whereas the fine bran (also called "shorts" in USA) which comes from the inner bran layers of wheat grain closer to aleurone and also includes the aleurone layer is obtained from reduction roll section of a roller flour mill.

\subsection{Rheological Characteristics}

2.2.1. Dough Making for Rheological Studies. For farinograph studies, as for the constant flour methods, $50 \mathrm{~g}$ of flour $(14 \%$ moisture basis) was used. The temperature of water was adjusted to $30 \pm 0.2^{\circ} \mathrm{C}$. The water bath $\left(30 \pm 0.2^{\circ} \mathrm{C}\right)$ was turned on at least one hour before using this instrument. After adding flour, the instrument was turned on for $1 \mathrm{~min}$ before the water was added from the burette into the right front corner of the bowl, continued mixing till the center of the graph arrived at the $500 \mathrm{BU}$ line. The amount of water needed to reach this height of $500 \mathrm{BU}$ was taken as farinograph water absorption (FWA).

For extensograph studies, $300 \mathrm{~g}$ of flour (14\% moisture basis) and $6 \mathrm{~g}$ salt (dissolved in water) were used in a large $300 \mathrm{~g}$ bowl on the farinograph instrument. Using $2 \%$ less water than the FWA to compensate for the use of salt, dough was mixed to arrive at the peak height of dough formation. At the end of mixing, $150 \pm 0.1 \mathrm{~g}$ of dough was scaled, gave 20 revolutions in the extensograph rounder unit, and then carefully shaped into a cylinder on the shaping unit. This cylinder was loaded into the lightly greased dough holder, clamped with the holder pins. Three cylinders so obtained were stored in the humidity chamber of the extensograph till testing was done for extensibility and resistance to extension at 45, 90, and $135 \mathrm{~min}$ of resting. After each stretching, dough was made into a cylinder the same way as explained above.

The replacement levels of white flour with coarse bran, fine bran, wheat germ, and psyllium husk were finalized after a number of preliminary studies in Hobart dough mixer for obtaining optimum dough with subjectively feeling of the dough quality. Levels higher than this resulted in detrimental dough quality with a very weak structure that was found not suitable for good baking performance.

2.2.2. Brabender Farinograph. Farinograph tests for wheat flour and various optimized blends containing raw wheat germ, bran fractions, and psyllium were conducted using a Brabender Farinograph (CW Brabender Co., Germany) equipped with a $50 \mathrm{~g}$ stainless-steel bowl. The constant flour weight procedure was used for obtaining farinograms, as per the AACC Method 54-21 [10]. The volume of water required to produce a curve with a maximum resistance centered on the 500-BU line was recorded as the farinograph water absorption (FWA). Brabender Farinograph (Brabender, Germany) was used to measure these properties of wheat flour dough (dough development time, peak time, tolerance index, stability, time to breakdown, valorimeter value).

2.2.3. Brabender Extensograph. Extensograph tests for wheat flour and various optimized blends containing raw wheat germ, bran fractions, and psyllium were conducted using a Brabender Extensograph. Dough elasticity and extensibility assessed by Brabender Extensograph (Brabender, Germany) were used to obtain three measurements (resistance to extension, extensibility, area under the curve) as per the AACC Method 54-10 [10].

2.3. Statistical Analysis. All the chemical analyses are reported on moisture free basis. All the experimental data obtained were analyzed statistically for analysis of variance, for statistical significance using Duncan's New Multiple Range Test (SAS Program Windows Version 6.08), and inference reported at the appropriate places. Significance 


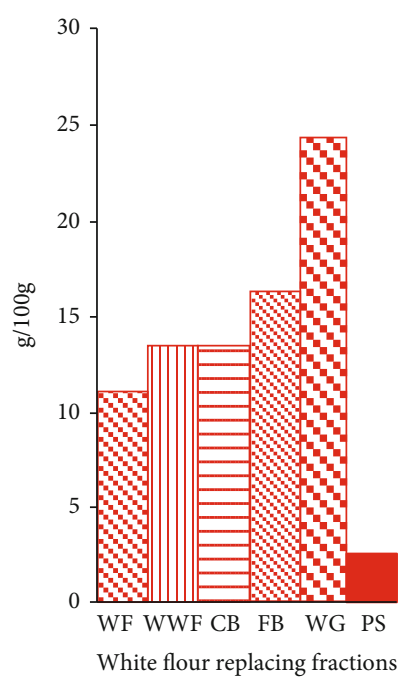

(a) Protein content

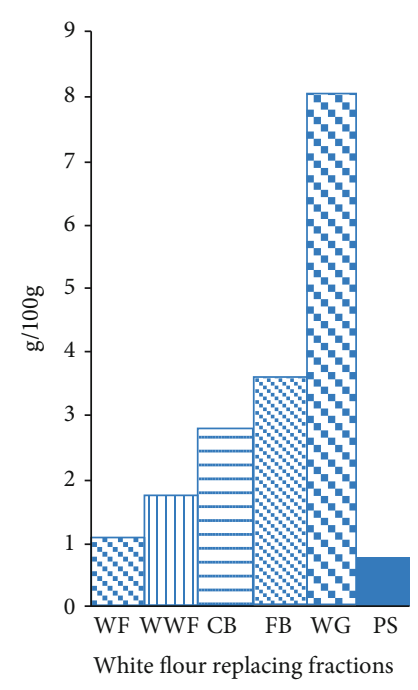

(b) Fat content

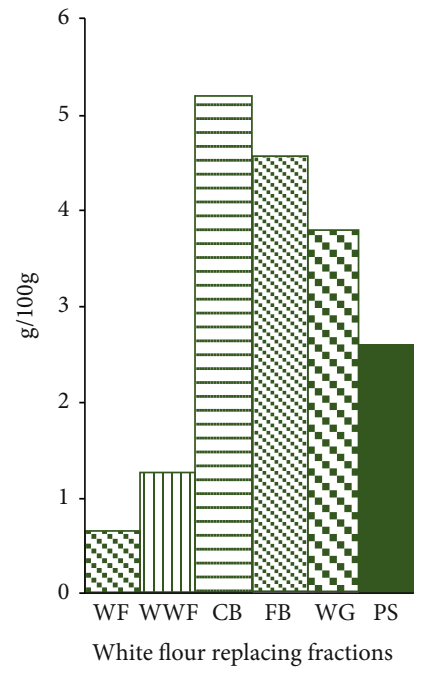

(c) Ash content

FIGURE 1: Protein, fat, and ash content of various fractions of wheat compared with the psyllium husk. Legend: WF: white flour; WWF: whole wheat flour; CB: coarse bran; FB: fine bran; WG: wheat germ; PS: psyllium husk.

TABLE 1: Farinograph characteristics of dough made from flour, bran fractions, and germ blends.

\begin{tabular}{|c|c|c|c|c|c|}
\hline Sample code & FWA \% & Peak time $(\mathrm{min})$ & Dough stability (min) & MTI (BU) & Valorimeter value \\
\hline WWF & $68.5^{\mathrm{a}}$ & $6.0^{\mathrm{a}}$ & $9.0^{\mathrm{a}}$ & $60^{\mathrm{a}}$ & $80^{\mathrm{a}}$ \\
\hline WF & $64.5^{\mathrm{b}}$ & $8.0^{\mathrm{b}}$ & $16.5^{\mathrm{b}}$ & $25^{\mathrm{b}}$ & $95^{\mathrm{b}}$ \\
\hline $\mathrm{WF}+10 \%$ fine bran & $64.0^{\mathrm{b}}$ & $7.0^{\mathrm{b}}$ & $9.0^{c}$ & $40^{\mathrm{c}}$ & $81^{\mathrm{c}}$ \\
\hline $\mathrm{WF}+20 \%$ fine bran & $67.0^{\mathrm{c}}$ & $5.5^{\mathrm{c}}$ & $8.0^{c}$ & $60^{\mathrm{d}}$ & $79^{c}$ \\
\hline $\mathrm{WF}+10 \%$ coarse bran & $64.0^{\mathrm{b}}$ & $7.0^{\mathrm{b}}$ & $16.0^{\mathrm{b}}$ & $5^{\mathrm{b}}$ & $95^{\mathrm{b}}$ \\
\hline $\mathrm{WF}+20 \%$ coarse bran & $67.0^{\mathrm{c}}$ & $6.0^{\mathrm{a}}$ & $17.0^{\mathrm{b}}$ & $15^{\mathrm{b}}$ & $95^{\mathrm{b}}$ \\
\hline $\mathrm{WF}+10 \%$ wheat germ & $61.0^{\mathrm{d}}$ & $4.0^{\mathrm{d}}$ & $3.5^{\mathrm{d}}$ & $135^{\mathrm{e}}$ & $60^{\mathrm{d}}$ \\
\hline $\mathrm{WF}+20 \%$ wheat germ & $60.0^{\mathrm{d}}$ & $4.5^{\mathrm{d}}$ & $3.5^{\mathrm{d}}$ & $205^{\mathrm{f}}$ & $58^{\mathrm{d}}$ \\
\hline
\end{tabular}

FWA: Farinograph water absorption; BU: Brabender units; MTI: mixing tolerance index: WWF: whole wheat flour; WF: white flour. Mean values $(N=3)$ with same superscripts for any parameter do not differ significantly in a column $(P=0.05)$.

was accepted at the $P=0.05$ level. For most of the results, the mean values with standard deviations are reported in the tables.

The reagents used in the chemical analyses of this work were of analytical grade.

\section{Results and Discussion}

3.1. Chemical Composition. Whole wheat flour, white flour, coarse bran, fine bran, psyllium, and germ used in this study were analyzed for crude protein, crude lipids, and ash contents. The proximate analyses (\% dry basis) for WF, WWF, $\mathrm{CB}, \mathrm{FB}, \mathrm{WG}$, and PS have been presented in Figure 1. The ash, protein, and fat contents of flour, bran, and germ were closer to those reported in the literature [11]. Bran fractions and raw wheat germ were found to be rich in minerals and protein. As expected, the ash content was the lowest in white flour $(0.64 \%)$ but was higher in bran and germ fractions. The protein contents of raw wheat germ were the highest (24.24\%) as compared with psyllium, which had the lowest value $(2.53 \%)$. The wheat germ, as expected, was especially richer in fat $(7.99 \%)$ contents. The higher fat and protein contents of fine bran samples compared with coarse bran may be due to the inclusion of layer and the germ, because most of these two components end up in these fine bran aleurone mill fractions during the normal wheat milling process. Unfortunately, during white flour production, coarse and fine bran as well as the germ are separated into mill fractions which constitute what is called as mill feed, and it goes into cattle feed manufacture.

3.2. Farinograph Characteristics. WF, WWF, and various combinations with coarse bran, fine bran, and psyllium were evaluated for dough rheology using a Brabender Farinograph and Brabender Extensograph, and the results are presented in Tables 1-12. Farinograph is a commonly used instrument in the laboratory for obtaining correct water absorption values for reaching the required dough consistency for producing good quality baked goods. FWA values were higher for WWF than for WF (Table 1). FWA increased progressively with increases in the addition of coarse bran as well as fine bran fractions to the WF. With the addition of fine bran as well as course bran, FWA values increased significantly at $20 \%$ level (Table 1). At $10 \%$ addition of these bran factions, 
TABLE 2: Farinograph characteristics of dough made from flour and psyllium blends.

\begin{tabular}{|c|c|c|c|c|c|}
\hline Sample code & FWA \% & Peak time (min) & Dough stability (min) & MTI (BU) & Valorimeter value \\
\hline WF & $64.5^{\mathrm{a}}$ & $8.0^{\mathrm{a}}$ & $16.5^{\mathrm{a}}$ & $25^{\mathrm{a}}$ & $95^{\mathrm{a}}$ \\
\hline $\mathrm{WF}+1 \%$ psyllium & $67.0^{\mathrm{b}}$ & $11.5^{\mathrm{b}}$ & $13.5^{\mathrm{b}}$ & $10^{\mathrm{a}}$ & $94^{\mathrm{a}}$ \\
\hline $\mathrm{WF}+2 \%$ psyllium & $71.5^{\mathrm{c}}$ & $11.0^{\mathrm{b}}$ & $14.0^{\mathrm{b}}$ & $25^{\mathrm{a}}$ & $94^{\mathrm{a}}$ \\
\hline $\mathrm{WF}+3 \%$ psyllium & $76.0^{\mathrm{d}}$ & $11.0^{\mathrm{b}}$ & $13.0^{\mathrm{b}}$ & $10^{\mathrm{a}}$ & $94^{\mathrm{a}}$ \\
\hline $\mathrm{WF}+4 \%$ psyllium & $80.0^{\mathrm{e}}$ & $11.0^{\mathrm{b}}$ & $11.0^{\mathrm{b}}$ & $30^{\mathrm{a}}$ & $94^{\mathrm{a}}$ \\
\hline $\mathrm{WF}+5 \%$ psyllium & $84.0^{\mathrm{f}}$ & $11.0^{\mathrm{b}}$ & $12.0^{\mathrm{b}}$ & $20^{\mathrm{a}}$ & $94^{\mathrm{a}}$ \\
\hline
\end{tabular}

FWA: Farinograph water absorption; BU: Brabender units; MTI: mixing tolerance index; WF: white flour. Mean values $(N=3)$ with same superscripts for any parameter do not differ significantly in a column $(P=0.05)$.

TABle 3: Farinograph characteristics of dough made from flour, fine bran, and psyllium blends.

\begin{tabular}{lccccc}
\hline Sample code & FWA \% & Peak time (min) & Dough stability (min) & MTI (BU) & Valorimeter value \\
\hline WF & $64.5^{\mathrm{a}}$ & $8.0^{\mathrm{a}}$ & $16.5^{\mathrm{a}}$ & $25^{\mathrm{a}}$ & $95^{\mathrm{a}}$ \\
WF+10\% fine bran+1\% psyllium & $67.5^{\mathrm{b}}$ & $6.5^{\mathrm{b}}$ & $10.5^{\mathrm{b}}$ & $45^{\mathrm{a}}$ & $87^{\mathrm{b}}$ \\
WF+10\% fine bran +2\% psyllium & $72.0^{\mathrm{c}}$ & $9.0^{\mathrm{ac}}$ & $10.0^{\mathrm{b}}$ & $55^{\mathrm{a}}$ & $87^{\mathrm{b}}$ \\
WF+10\% fine bran +3\% psyllium & $81.0^{\mathrm{d}}$ & $9.0^{\mathrm{ac}}$ & $11.0^{\mathrm{b}}$ & $45^{\mathrm{a}}$ & $87^{\mathrm{b}}$ \\
WF+10\% fine bran +4\% psyllium & $86.0^{\mathrm{e}}$ & $10.0^{\mathrm{c}}$ & $12.0^{\mathrm{a}}$ & $40^{\mathrm{a}}$ & $25^{\mathrm{a}}$ \\
WF+10\% fine bran +5\% psyllium & $93.5^{\mathrm{f}}$ & $10.0^{\mathrm{c}}$ & $13.5^{\mathrm{a}}$ & $94^{\mathrm{a}}$ \\
\hline
\end{tabular}

FWA: Farinograph water absorption; BU: Brabender units; MTI: mixing tolerance index; WF: white flour. Mean values $(N=3)$ with same superscripts for any parameter do not differ significantly in a column $(P=0.05)$.

TABLE 4: Farinograph characteristics of dough made from flour, fine bran, and psyllium blends.

\begin{tabular}{|c|c|c|c|c|c|}
\hline Sample code & FWA \% & Peak time $(\mathrm{min})$ & Dough stability (min) & MTI (BU) & Valorimeter value \\
\hline WF & $64.5^{\mathrm{a}}$ & $8.0^{\mathrm{a}}$ & $16.5^{\mathrm{a}}$ & $25^{\mathrm{a}}$ & $95^{\mathrm{a}}$ \\
\hline $\mathrm{WF}+20 \%$ fine bran $+1 \%$ psyllium & $70.0^{\mathrm{b}}$ & $6.0^{\mathrm{b}}$ & $9.5^{\mathrm{b}}$ & $75^{\mathrm{b}}$ & $84^{\mathrm{b}}$ \\
\hline $\mathrm{WF}+20 \%$ fine bran $+2 \%$ psyllium & $76.0^{c}$ & $7.0^{\mathrm{c}}$ & $9.0^{\mathrm{b}}$ & $70^{\mathrm{b}}$ & $85^{\mathrm{b}}$ \\
\hline $\mathrm{WF}+20 \%$ fine bran $+3 \%$ psyllium & $84.0^{\mathrm{d}}$ & $8.5^{\mathrm{a}}$ & $9.0^{\mathrm{b}}$ & $70^{\mathrm{b}}$ & $85^{\mathrm{b}}$ \\
\hline $\mathrm{WF}+20 \%$ fine bran $+4 \%$ psyllium & $89.0^{\mathrm{e}}$ & $9.5^{\mathrm{d}}$ & $10.0^{c}$ & $55^{\mathrm{c}}$ & $91^{\mathrm{a}}$ \\
\hline $\mathrm{WF}+20 \%$ fine bran $+5 \%$ psyllium & $92.0^{\mathrm{f}}$ & $10.5^{\mathrm{e}}$ & $12.0^{\mathrm{d}}$ & $45^{\mathrm{c}}$ & $93^{\mathrm{a}}$ \\
\hline
\end{tabular}

FWA: Farinograph water absorption; BU: Brabender units; MTI: mixing tolerance index; WF: white flour. Mean values $(N=3)$ with same superscripts for any parameter do not differ significantly in a column $(P=0.05)$.

TABLE 5: Farinograph characteristics of dough made from flour, coarse bran, and psyllium blends.

\begin{tabular}{|c|c|c|c|c|c|}
\hline Sample code & FWA \% & Peak time (min) & Dough stability (min) & MTI (BU) & Valorimeter value \\
\hline WF & $64.5^{\mathrm{a}}$ & $8.0^{\mathrm{a}}$ & $16.5^{\mathrm{a}}$ & $25^{\mathrm{a}}$ & $95^{\mathrm{a}}$ \\
\hline $\mathrm{WF}+10 \%$ coarse bran $+1 \%$ psyllium & $75.0^{\mathrm{b}}$ & $10.5^{\mathrm{b}}$ & $13.0^{\mathrm{b}}$ & $30^{\mathrm{b}}$ & $94^{\mathrm{a}}$ \\
\hline $\mathrm{WF}+10 \%$ coarse bran $+2 \%$ psyllium & $82.0^{\mathrm{c}}$ & $10.0^{\mathrm{b}}$ & $12.0^{\mathrm{b}}$ & $30^{\mathrm{b}}$ & $94^{\mathrm{a}}$ \\
\hline $\mathrm{WF}+10 \%$ coarse bran $+3 \%$ psyllium & $90.0^{\mathrm{d}}$ & $13.0^{\mathrm{c}}$ & $15.0^{\mathrm{b}}$ & $20^{\mathrm{a}}$ & $94^{\mathrm{a}}$ \\
\hline $\mathrm{WF}+10 \%$ coarse bran $+4 \%$ psyllium & $95.0^{\mathrm{e}}$ & $12.5^{\mathrm{c}}$ & $11.5^{\mathrm{b}}$ & $30^{\mathrm{b}}$ & $94^{\mathrm{a}}$ \\
\hline $\mathrm{WF}+10 \%$ coarse bran $+5 \%$ psyllium & $100.0^{\mathrm{f}}$ & $13.5^{\mathrm{c}}$ & $10.5^{\mathrm{b}}$ & $35^{\mathrm{b}}$ & $94^{\mathrm{a}}$ \\
\hline
\end{tabular}

FWA: Farinograph water absorption; BU: Brabender units; MTI: mixing tolerance index; WF: white flour. Mean values $(N=3)$ with same superscripts for any parameter do not differ significantly in a column $(P=0.05)$.

the additional water picked up got neutralized by the dilution of the gluten content in WF. Regardless of the level of bran addition, it is known to adversely affect the formation of the proper structure during mixing of dough [12].

As the bran particles have finer size, their surface area increases leading to higher water absorption. Similar findings have also been reported by Pauly et al. [13]. The FWA for WF in our study is almost identical to the one reported by Hem- ery et al. [14] but the FWA for WWF in our study is slightly lower than the one reported by them. This may possibly be due to the differences in the quality and quantity of gluten proteins or the particle size of flour or bran particles. The ultrafine milling of flour to 18.36 to $57.96 \mu \mathrm{m}$ particle size has been reported to result in higher Farinograph water absorption from 59.1 to $72.9 \%$ [15]. Another important factor that affects the FWA is the amount of the damaged 
TABLE 6: Farinograph characteristics of dough made from flour, coarse bran, and psyllium blends.

\begin{tabular}{|c|c|c|c|c|c|}
\hline Sample code & FWA \% & Peak time (min) & Dough stability (min) & MTI (BU) & Valorimeter value \\
\hline WF & $64.5^{\mathrm{a}}$ & $8.0^{\mathrm{a}}$ & $16.5^{\mathrm{a}}$ & $25^{\mathrm{a}}$ & $95^{\mathrm{a}}$ \\
\hline $\mathrm{WF}+20 \%$ coarse bran $+1 \%$ psyllium & $78.0^{\mathrm{b}}$ & $8.0^{\mathrm{a}}$ & $14.0^{\mathrm{a}}$ & $5^{\mathrm{b}}$ & $94^{\mathrm{a}}$ \\
\hline $\mathrm{WF}+20 \%$ coarse bran $+2 \%$ psyllium & $84.0^{\mathrm{c}}$ & $9.5^{\mathrm{b}}$ & $13.0^{\mathrm{a}}$ & $5^{\mathrm{b}}$ & $94^{\mathrm{a}}$ \\
\hline $\mathrm{WF}+20 \%$ coarse bran $+3 \%$ psyllium & $89.0^{\mathrm{d}}$ & $10.0^{\mathrm{b}}$ & $13.0^{\mathrm{a}}$ & $10^{\mathrm{b}}$ & $94^{\mathrm{a}}$ \\
\hline $\mathrm{WF}+20 \%$ coarse bran $+4 \%$ psyllium & $94.0^{\mathrm{e}}$ & $10.0^{\mathrm{b}}$ & $13.0^{\mathrm{a}}$ & $10^{\mathrm{b}}$ & $94^{\mathrm{a}}$ \\
\hline $\mathrm{WF}+20 \%$ coarse bran $+5 \%$ psyllium & $99.0^{\mathrm{f}}$ & $13.5^{\mathrm{c}}$ & $13.0^{\mathrm{a}}$ & $5^{\mathrm{b}}$ & $94^{\mathrm{a}}$ \\
\hline
\end{tabular}

FWA: Farinograph water absorption; BU: Brabender units; MTI: mixing tolerance index; WF: white flour. Mean values $(N=3)$ with same superscripts for any parameter do not differ significantly in a column $(P=0.05)$.

TABLE 7: Extensograph characteristics of dough made from flour, germ, bran fractions, and psyllium blends.

\begin{tabular}{|c|c|c|c|c|c|c|c|c|c|}
\hline \multirow{2}{*}{ Sample code } & \multicolumn{3}{|c|}{ Extensibility $(\mathrm{cm})$} & \multicolumn{3}{|c|}{ Resistance to extension $(\mathrm{cm})$} & \multicolumn{3}{|c|}{ Area under the curve $\left(\mathrm{cm}^{2}\right)$} \\
\hline & 45 & 90 & 135 & 45 & 90 & 135 & 45 & 90 & 135 \\
\hline Whole wheat flour (WWF) & $16.7_{\mathrm{f}}^{\mathrm{a}}$ & $14.9_{\mathrm{fg}}$ & $13.3_{\mathrm{g}}$ & $7.2_{\mathrm{f}}^{\mathrm{a}}$ & $9.5_{\mathrm{g}}$ & $11.3_{\mathrm{h}}$ & $83.5_{\mathrm{f}}^{\mathrm{a}}$ & $100.5_{\mathrm{g}}$ & $104.9_{\mathrm{g}}$ \\
\hline White flour (WF) & $25.9^{b}{ }_{f}$ & $24.4_{\mathrm{g}}$ & $23.5_{\mathrm{fg}}$ & $11.9_{\mathrm{f}}^{\mathrm{b}}$ & $15.2_{\mathrm{g}}$ & $15.4_{\mathrm{g}}$ & $213.0^{b}$ & $254.6_{\mathrm{g}}$ & $247.7_{\mathrm{g}}$ \\
\hline $\mathrm{WF}+10 \%$ fine bran & $19.2_{\mathrm{f}}^{\mathrm{c}}$ & $18.4_{\mathrm{f}}$ & $17.5_{\mathrm{g}}$ & $10.8_{\mathrm{f}}^{\mathrm{bc}}$ & $13.4_{\mathrm{g}}$ & $15.3_{\mathrm{h}}$ & $150.3^{c}{ }_{j}$ & $177.6_{\mathrm{k}}$ & $185.4_{\mathrm{k}}$ \\
\hline $\mathrm{WF}+20 \%$ fine bran & $14.9_{\mathrm{f}}^{\mathrm{d}}$ & $14.1_{\mathrm{f}}$ & $14.1_{\mathrm{f}}$ & $10.1_{\mathrm{f}}^{\mathrm{c}}$ & $12.8_{\mathrm{g}}$ & $13.2_{\mathrm{g}}$ & $119.7^{\mathrm{d}}{ }_{\mathrm{j}}$ & $133.5_{\mathrm{jk}}$ & $137.2_{\mathrm{k}}$ \\
\hline $\mathrm{WF}+10 \%$ coarse bran & $16.6_{\mathrm{f}}^{\mathrm{e}}$ & $15.0_{\mathrm{f}}$ & $15.3_{\mathrm{f}}$ & $14.2_{\mathrm{f}}^{\mathrm{d}}$ & $17.8_{\mathrm{g}}$ & $18.6_{\mathrm{g}}$ & $174.4^{\mathrm{e}}{ }_{j}$ & $191.4_{\mathrm{jk}}$ & $202.9_{\mathrm{k}}$ \\
\hline $\mathrm{WF}+20 \%$ coarse bran & $11.9_{\mathrm{f}}^{\mathrm{m}}$ & $11.2_{\mathrm{f}}$ & $9.7_{\mathrm{g}}$ & $13.1_{\mathrm{f}}^{\mathrm{bd}}$ & $16.4_{\mathrm{g}}$ & $18.6_{\mathrm{h}}$ & $130.6_{j}^{f}$ & $134.8_{\mathrm{k}}$ & $127.5_{\mathrm{jk}}$ \\
\hline $\mathrm{WF}+10 \%$ wheat germ & $24.6_{\mathrm{f}}^{\mathrm{b}}$ & $21.4_{\mathrm{g}}$ & $20.2_{\mathrm{h}}$ & $3.9_{\mathrm{g}}^{\mathrm{e}}$ & $4.0_{\mathrm{g}}$ & $4.6_{\mathrm{g}}$ & $175.5^{\mathrm{g}}{ }_{\mathrm{j}}$ & $177.1_{\mathrm{j}}$ & $182.3_{j}$ \\
\hline $\mathrm{WF}+20 \%$ wheat germ & $22.7_{\mathrm{f}}^{\mathrm{n}}$ & $21.3_{\mathrm{f}}$ & $20.8_{\mathrm{f}}$ & $4.0_{\mathrm{g}}^{\mathrm{e}}$ & $4.2_{\mathrm{gh}}$ & $4.7_{\mathrm{h}}$ & $179.5^{\mathrm{g}}{ }_{\mathrm{j}}$ & $182.5_{j}$ & $185.2_{\mathrm{j}}$ \\
\hline
\end{tabular}

Mean values $(N=3)$ with same superscripts for any parameter do not differ significantly in a column $(P=0.05)$. Mean values with same subscripts for any parameter (i.e., extensibility, resistance to extension, or area under the curve) at 45,90 , and 135 minutes do not differ significantly in a row $(P=0.05)$.

TABLE 8: Extensograph characteristics of dough made from flour and psyllium blends.

\begin{tabular}{|c|c|c|c|c|c|c|c|c|c|}
\hline \multirow{2}{*}{ Sample code } & \multicolumn{3}{|c|}{ Extensibility $(\mathrm{cm})$} & \multicolumn{3}{|c|}{ Resistance to extension $(\mathrm{cm})$} & \multicolumn{3}{|c|}{ Area under the curve $\left(\mathrm{cm}^{2}\right)$} \\
\hline & 45 & 90 & 135 & 45 & 90 & 135 & 45 & 90 & 135 \\
\hline White flour (WF) & $25.9^{a}{ }_{f}$ & $24.4_{\mathrm{g}}$ & $23.5_{\mathrm{g}}$ & $11.9^{a}{ }_{f}$ & $15.2_{\mathrm{g}}$ & $15.4_{\mathrm{g}}$ & $213.0^{a}{ }_{f}$ & $254.6_{\mathrm{g}}$ & $247.7_{\mathrm{g}}$ \\
\hline $\mathrm{WF}+1 \%$ psyllium & $18.1_{\mathrm{f}}^{\mathrm{b}}$ & $17.4_{\mathrm{f}}$ & $17.6_{\mathrm{f}}^{\circ}$ & $13.8_{\mathrm{f}}^{\mathrm{b}}$ & $18.2_{\mathrm{g}}^{\circ}$ & $18.2_{\mathrm{g}}^{\circ}$ & $175.7_{f}^{a}$ & $227.8_{\mathrm{g}}$ & $241.1_{\mathrm{g}}$ \\
\hline $\mathrm{WF}+2 \%$ psyllium & $16.6^{\mathrm{b}}{ }_{\mathrm{f}}$ & $15.9_{\mathrm{f}}$ & $15.6_{\mathrm{f}}$ & $15.6_{\mathrm{f}}^{\mathrm{b}}$ & $18.1_{\mathrm{g}}^{\circ}$ & $18.3_{\mathrm{g}}^{\circ}$ & $185.4_{f}^{\mathrm{ab}}$ & $215.1^{\circ}$ & $211.3_{\mathrm{g}}$ \\
\hline $\mathrm{WF}+3 \%$ psyllium & $16.3^{\mathrm{b}}{ }_{\mathrm{f}}$ & $17.4_{\mathrm{f}}$ & $16.0_{\mathrm{f}}$ & $13.8_{\mathrm{f}}^{\mathrm{b}}$ & $18.2_{\mathrm{g}}^{\circ}$ & $18.2_{\mathrm{g}}^{\circ}$ & $180.6_{f}^{\mathrm{ab}}$ & $241.0_{\mathrm{g}}$ & $224.9_{\mathrm{g}}^{\circ}$ \\
\hline $\mathrm{WF}+4 \%$ psyllium & $12.6_{\mathrm{f}}^{\mathrm{c}}$ & $9.8_{\mathrm{g}}$ & $8.8_{\mathrm{g}}$ & $16.7_{\mathrm{f}}^{\mathrm{b}}$ & $18.2_{\mathrm{g}}^{\circ}$ & $18.2_{\mathrm{g}}^{\circ}$ & $229.6_{f}^{a}$ & $234.6_{\mathrm{f}}^{\circ}$ & $256.3_{\mathrm{f}}$ \\
\hline $\mathrm{WF}+5 \%$ psyllium & $14.1_{\mathrm{f}}^{\mathrm{d}}$ & $13.4_{\mathrm{f}}$ & $10.2_{\mathrm{f}}$ & $17.1_{\mathrm{f}}^{\mathrm{b}}$ & $18.2_{\mathrm{f}}^{\circ}$ & $18.3_{\mathrm{f}}$ & $188.8^{\mathrm{b}}{ }_{\mathrm{f}}$ & $196.6_{\mathrm{f}}$ & $177.6_{f}$ \\
\hline
\end{tabular}

Mean values $(N=3)$ with same superscripts for any parameter do not differ significantly in a column $(P=0.05)$. Mean values with same subscripts for any parameter (i.e., extensibility, resistance to extension, or area under the curve) at 45,90 , and 135 minutes do not differ significantly in a row $(P=0.05)$.

TABLE 9: Extensograph characteristics of dough made from flour, fine bran, and psyllium blends.

\begin{tabular}{|c|c|c|c|c|c|c|c|c|c|}
\hline \multirow{2}{*}{ Sample code } & \multicolumn{3}{|c|}{ Extensibility $(\mathrm{cm})$} & \multicolumn{3}{|c|}{ Resistance to extension (cm) } & \multicolumn{3}{|c|}{ Area under the curve $\left(\mathrm{cm}^{2}\right)$} \\
\hline & 45 & 90 & 135 & 45 & 90 & 135 & 45 & 90 & 135 \\
\hline White flour (WF) & $25.9^{a}{ }_{f}$ & $24.4_{\mathrm{g}}$ & $23.5_{\mathrm{g}}$ & $11.9^{a}{ }_{f}$ & $15.2_{\mathrm{g}}$ & $15.4_{\mathrm{g}}$ & $213.0_{f}^{a}$ & $254.6 \mathrm{~g}$ & $247.7 \mathrm{~g}$ \\
\hline $\mathrm{WF}+10 \%$ fine bran $+1 \%$ psyllium & $17.6_{f}^{b}$ & $16.4_{\mathrm{g}}$ & $16.6_{\mathrm{g}}$ & $9.7_{\mathrm{f}}^{\mathrm{b}}$ & $12.2_{\mathrm{g}}$ & $13.3_{\mathrm{h}}$ & $137.9_{\mathrm{f}}^{\mathrm{b}}$ & $156.2_{\mathrm{g}}^{\circ}$ & $171.3_{\mathrm{g}}^{\circ}$ \\
\hline $\mathrm{WF}+10 \%$ fine bran $+2 \%$ psyllium & $15.1_{f}^{c d}$ & $14.0_{\mathrm{f}}$ & $13.5_{\mathrm{f}}$ & $13.5_{\mathrm{f}}^{\mathrm{a}}$ & $16.0_{\mathrm{g}}$ & $18.0_{\mathrm{h}}$ & $149.4_{\mathrm{f}}^{\mathrm{b}}$ & $164.2_{\mathrm{fg}}^{\circ}$ & $169.9_{\mathrm{g}}^{\circ}$ \\
\hline $\mathrm{WF}+10 \%$ fine bran $+3 \%$ psyllium & $15.7_{\mathrm{f}}^{\mathrm{c}}$ & $14.9_{\mathrm{f}}$ & $14.0_{\mathrm{f}}$ & $10.5^{\mathrm{ab}}{ }_{\mathrm{f}}$ & $14.0_{\mathrm{g}}$ & $15.7_{\mathrm{h}}$ & $130.2_{\mathrm{f}}^{\mathrm{b}}$ & $157.6_{g}^{\circ}$ & $162.1_{\mathrm{g}}$ \\
\hline $\mathrm{WF}+10 \%$ fine bran $+4 \%$ psyllium & $14.7_{\mathrm{f}}^{\mathrm{d}}$ & $13.0_{\mathrm{f}}$ & $12.7_{\mathrm{f}}$ & $10.9_{\mathrm{f}}^{\mathrm{a}}$ & $16.0_{\mathrm{g}}$ & $18.2_{\mathrm{h}}$ & $125.3_{\mathrm{f}}^{\mathrm{b}}$ & $149.1_{\mathrm{g}}$ & $167.0_{\mathrm{g}}$ \\
\hline WF $+10 \%$ fine bran $+5 \%$ psyllium & $12.6_{\mathrm{f}}^{\mathrm{e}}$ & $12.0_{\mathrm{f}}$ & $11.9_{\mathrm{f}}$ & $12.7_{\mathrm{f}}^{\mathrm{a}}$ & $16.5_{\mathrm{g}}$ & $18.2_{\mathrm{h}}$ & $127.1_{\mathrm{f}}^{\mathrm{b}}$ & $150.0 \mathrm{~g}$ & $159.7_{\mathrm{g}}$ \\
\hline
\end{tabular}

WWF: whole wheat flour; WF: white flour. Mean values $(N=3)$ with same superscripts for any parameter do not differ significantly in a column $(P=0.05)$. Mean values with same subscripts for any parameter (i.e., extensibility, resistance to extension, or area under the curve) at 45,90 , and 135 minutes do not differ significantly in a row $(P=0.05)$. 
TABLE 10: Extensograph characteristics of dough made from flour, fine bran, and psyllium blends.

\begin{tabular}{|c|c|c|c|c|c|c|c|c|c|}
\hline \multirow{2}{*}{ Sample code } & \multicolumn{3}{|c|}{ Extensibility $(\mathrm{cm})$} & \multicolumn{3}{|c|}{$\begin{array}{l}\text { Resistance to extension } \\
\qquad(\mathrm{cm})\end{array}$} & \multicolumn{3}{|c|}{ Area under the curve $\left(\mathrm{cm}^{2}\right)$} \\
\hline & 45 & 90 & 135 & 45 & 90 & 135 & 45 & 90 & 135 \\
\hline White flour (WF) & $25.9^{a}$ & $24.4_{\mathrm{g}}$ & $23.5_{\mathrm{g}}$ & $11.9^{\mathrm{a}}$ & $15.2_{\mathrm{g}}$ & $15.4_{\mathrm{g}}$ & $213.0^{\mathrm{a}}$ & $254.6_{\mathrm{g}}$ & $247.7_{\mathrm{g}}$ \\
\hline $\mathrm{WF}+20 \%$ fine bran $+1 \%$ psyllium & $13.7^{\mathrm{b}}$ & $13.6_{\mathrm{f}}^{\circ}$ & $13.3_{\mathrm{f}}$ & $10.0^{\mathrm{a}}{ }_{\mathrm{f}}$ & $12.5_{\mathrm{g}}^{\circ}$ & $14.4_{\mathrm{h}}^{\circ}$ & $107.5_{\mathrm{f}}^{\mathrm{b}}$ & $134.2_{\mathrm{g}}^{\mathrm{b}}$ & $144.2_{\mathrm{g}}^{\circ}$ \\
\hline $\mathrm{WF}+20 \%$ fine bran $+2 \%$ psyllium & $13.5^{\mathrm{b}}$ & $13.1_{\mathrm{fg}}$ & $12.1_{\mathrm{g}}$ & $9.4_{f}^{\mathrm{a}}$ & $12.6_{\mathrm{g}}^{\circ}$ & $15.0_{\mathrm{h}}$ & $102.7^{b c}$ & $126.0_{\mathrm{g}}^{\mathrm{g}}$ & $138.1_{\mathrm{g}}^{\circ}$ \\
\hline $\mathrm{WF}+20 \%$ fine bran $+3 \%$ psyllium & $11.4_{\mathrm{f}}^{\mathrm{c}}$ & $11.2_{\mathrm{f}}$ & $10.6_{\mathrm{f}}$ & $11.3_{\mathrm{f}}^{\mathrm{a}}$ & $15.1_{\mathrm{g}}$ & $17.5_{\mathrm{h}}$ & $101.6_{f}^{b c}$ & $124.9_{\mathrm{g}}^{\mathrm{g}}$ & $131.7_{\mathrm{g}}^{\circ}$ \\
\hline $\mathrm{WF}+20 \%$ fine bran $+4 \%$ psyllium & $12.3_{\mathrm{f}}^{\mathrm{c}}$ & $11.7_{\mathrm{f}}$ & $11.0_{\mathrm{f}}$ & $10.1_{\mathrm{f}}^{\mathrm{a}}$ & $12.8_{\mathrm{g}}^{\circ}$ & $16.1_{\mathrm{h}}$ & $94.1_{f}^{\mathrm{cd}}$ & $110.4_{\mathrm{g}}^{\mathrm{g}}$ & $126.1_{\mathrm{g}}^{\circ}$ \\
\hline $\mathrm{WF}+20 \%$ fine bran $+5 \%$ psyllium & $11.2^{\mathrm{d}}{ }_{\mathrm{f}}$ & $9.3_{\mathrm{g}}$ & $8.9_{\mathrm{g}}$ & $10.9_{\mathrm{f}}^{\mathrm{a}}$ & $14.7_{\mathrm{g}}^{\circ}$ & $16.2_{\mathrm{h}}$ & $83.0_{\mathrm{f}}^{\mathrm{d}}$ & $103.8_{\mathrm{g}}$ & $98.7_{\mathrm{g}}^{\circ}$ \\
\hline
\end{tabular}

Mean values $(N=3)$ with same superscripts for any parameter do not differ significantly in a column $(P=0.05)$. Mean values with same subscripts for any parameter (i.e., extensibility, resistance to extension, or area under the curve) at 45,90 , and 135 minutes do not differ significantly in a row $(P=0.05)$.

TABLE 11: Extensograph characteristics of dough made from flour, coarse bran, and psyllium blends.

\begin{tabular}{|c|c|c|c|c|c|c|c|c|c|}
\hline \multirow{2}{*}{ Sample code } & \multicolumn{3}{|c|}{ Extensibility $(\mathrm{cm})$} & \multicolumn{3}{|c|}{ Resistance to extension $(\mathrm{cm})$} & \multicolumn{3}{|c|}{ Area under the curve $\left(\mathrm{cm}^{2}\right)$} \\
\hline & 45 & 90 & 135 & 45 & 90 & 135 & 45 & 90 & 135 \\
\hline White flour (WF) & $25.9^{a}{ }_{f}$ & $24.4_{\mathrm{g}}$ & $23.5_{\mathrm{g}}$ & $11.9^{\mathrm{ab}}$ & $15.2 \mathrm{~g}$ & $15.4_{\mathrm{g}}$ & $213.0^{\mathrm{a}}$ & $254.6_{g}$ & $247.7_{\mathrm{g}}$ \\
\hline $\mathrm{WF}+10 \%$ coarse bran $+1 \%$ psyllium & $17.1_{\mathrm{f}}^{\mathrm{b}}$ & $16.2_{\mathrm{f}}$ & $14.7_{\mathrm{g}}$ & $11.3^{\mathrm{ab}}{ }_{\mathrm{f}}$ & $15.9_{\mathrm{g}}^{\circ}$ & $18.5_{\mathrm{h}}^{\circ}$ & $142.8_{\mathrm{f}}^{\mathrm{b}}$ & $177.7_{\mathrm{g}}^{\circ}$ & $190.2_{\mathrm{g}}$ \\
\hline $\mathrm{WF}+10 \%$ coarse bran $+2 \%$ psyllium & $17.4_{\mathrm{f}}^{\mathrm{b}}$ & $15.9_{\mathrm{g}}$ & $13.8_{\mathrm{h}}^{\mathrm{b}}$ & $9.3_{f}^{\mathrm{a}}$ & $13.9_{\mathrm{g}}^{\mathrm{g}}$ & $16.9_{h}$ & $121.2_{\mathrm{f}}^{\mathrm{c}}$ & $158.9_{\mathrm{g}}$ & $154.4_{\mathrm{g}}^{\mathrm{B}}$ \\
\hline $\mathrm{WF}+10 \%$ coarse bran $+3 \%$ psyllium & $16.2^{c}{ }_{f}$ & $13.6_{\mathrm{g}}$ & $12.4_{\mathrm{g}}$ & $8.4_{\mathrm{f}}^{\mathrm{ab}}$ & $16.4 \mathrm{~g}$ & $18.5_{\mathrm{g}}$ & $99.4_{\mathrm{f}}^{\mathrm{c}}$ & $147.6_{\mathrm{g}}$ & $140.6_{\mathrm{fg}}$ \\
\hline $\mathrm{WF}+10 \%$ coarse bran $+4 \%$ psyllium & $13.6^{\mathrm{d}}$ & $12.2_{\mathrm{f}}$ & $9.9 \mathrm{~g}$ & $12.8_{\mathrm{f}}^{\mathrm{ab}}$ & $18.4_{\mathrm{g}}$ & $18.7_{\mathrm{g}}$ & $126.8_{\mathrm{f}}^{\mathrm{c}}$ & $158.0_{\mathrm{g}}$ & $133.9_{\mathrm{fg}}$ \\
\hline $\mathrm{WF}+10 \%$ coarse bran $+5 \%$ psyllium & $13.2^{\mathrm{d}}{ }_{\mathrm{f}}$ & $10.5_{\mathrm{g}}$ & $9.3_{\mathrm{g}}$ & $13.7_{\mathrm{f}}^{\mathrm{b}}$ & $18.3_{\mathrm{g}}$ & $18.7_{\mathrm{g}}$ & $131.8_{\mathrm{f}}^{\mathrm{c}}$ & $132.6_{\mathrm{f}}$ & $122.7_{\mathrm{f}}$ \\
\hline
\end{tabular}

Mean values $(N=3)$ with same superscripts for any parameter do not differ significantly in a column $(P=0.05)$. Mean values with same subscripts for any parameter (i.e., extensibility, resistance to extension, or area under the curve) at 45,90 , and 135 minutes do not differ significantly in a row $(P=0.05)$.

TABLE 12: Extensograph characteristics of dough made from flour, coarse bran, and psyllium blends.

\begin{tabular}{|c|c|c|c|c|c|c|c|c|c|}
\hline \multirow{2}{*}{ Sample code } & \multicolumn{3}{|c|}{ Extensibility $(\mathrm{cm})$} & \multicolumn{3}{|c|}{$\begin{array}{l}\text { Resistance to extension } \\
\qquad(\mathrm{cm})\end{array}$} & \multicolumn{3}{|c|}{ Area under the curve $\left(\mathrm{cm}^{2}\right)$} \\
\hline & 45 & 90 & 135 & 45 & 90 & 135 & 45 & 90 & 135 \\
\hline White flour (WF) & $25.9_{\mathrm{f}}^{\mathrm{a}}$ & $24.4_{\mathrm{g}}$ & $23.5_{\mathrm{g}}$ & $11.9^{\mathrm{a}}$ & $15.2 \mathrm{~g}$ & $15.4 \mathrm{~g}$ & $213.0_{f}^{a}$ & $254.6_{\mathrm{g}}$ & $247.7_{\mathrm{g}}$ \\
\hline $\mathrm{WF}+20 \%$ coarse bran $+1 \%$ psyllium & $11.3_{\mathrm{f}}^{\mathrm{bc}}$ & $9.3_{\mathrm{g}}$ & $8.2_{\mathrm{g}}$ & $11.5_{\mathrm{f}}^{\mathrm{a}}$ & $18.0 \mathrm{~g}$ & $18.8 \mathrm{~g}$ & $95.5_{\mathrm{f}}^{\mathrm{b}}$ & $114.7_{\mathrm{f}}$ & $103.6_{\mathrm{f}}$ \\
\hline $\mathrm{WF}+20 \%$ coarse bran $+2 \%$ psyllium & $11.8_{\mathrm{f}}^{\mathrm{b}}$ & $10.6_{\mathrm{fg}}$ & $9.5_{\mathrm{g}}$ & $9.9_{\mathrm{f}}^{\mathrm{a}}$ & $16.5 \mathrm{~g}$ & $18.8_{\mathrm{h}}$ & $88.7_{\mathrm{f}}^{\mathrm{b}}$ & $122.1 \mathrm{~g}$ & $117.3 \mathrm{~g}$ \\
\hline $\mathrm{WF}+20 \%$ coarse bran $+3 \%$ psyllium & $11.3_{\mathrm{f}}^{\mathrm{b}}$ & $10.5_{\mathrm{fg}}^{\circ}$ & $9.9_{\mathrm{g}}$ & $10.7_{\mathrm{f}}^{\mathrm{a}}$ & $14.9_{\mathrm{g}}^{\circ}$ & $18.4_{\mathrm{h}}$ & $91.6^{\mathrm{b}}{ }_{\mathrm{f}}$ & $116.4_{\mathrm{g}}^{\circ}$ & $120.4_{\mathrm{g}}$ \\
\hline $\mathrm{WF}+20 \%$ coarse bran $+4 \%$ psyllium & $10.9_{\mathrm{f}}^{\mathrm{cd}}$ & $9.3_{\mathrm{g}}$ & $7.7_{\mathrm{h}}$ & $13.1_{\mathrm{f}}^{\mathrm{a}}$ & $18.1_{\mathrm{g}}^{\circ}$ & $18.7 \mathrm{~g}$ & $107.1_{\mathrm{f}}^{\mathrm{b}}$ & $114.6_{\mathrm{g}}^{\circ}$ & $110.9_{\mathrm{g}}^{\circ}$ \\
\hline $\mathrm{WF}+20 \%$ coarse bran $+5 \%$ psyllium & $9.9_{\mathrm{f}}^{\mathrm{d}}$ & $8.0_{\mathrm{g}}$ & $7.0_{\mathrm{h}}$ & $13.3_{\mathrm{f}}^{\mathrm{a}}$ & $17.4_{\mathrm{g}}$ & $18.2 \mathrm{~g}$ & $96.5^{\mathrm{b}}{ }_{\mathrm{f}}$ & $98.5_{f}$ & $88.8_{\mathrm{f}}$ \\
\hline
\end{tabular}

Mean values $(N=3)$ with same superscripts for any parameter do not differ significantly in a column $(P=0.05)$. Mean values with same subscripts for any parameter (i.e., extensibility, resistance to extension, or area under the curve) at 45,90 , and 135 minutes do not differ significantly in a row $(P=0.05)$.

starch content of a normally milled flour [16]. The grinding of starch granules to finer size increased the surface area, thus leading to higher water absorption values [17]. The addition of fine bran and coarse bran at $20 \%$ level significantly increased the FWA, peak time, dough stability, and valorimeter value (Table 1). The bran dietary fiber has a large numbers of hydroxyl groups which can bind tremendous amount water due to hydrogen bonding, much more than the major polymers present in wheat dough, i.e., gluten and starch [18-20]. The wheat germ being a rich source of glutathione, a known reducing agent, made the dough weaker during mixing; thus, the water absorption had to be reduced by nearly $4 \%$ than the WF to get an optimum dough consistency of $500 \mathrm{BU}$ (Table 1).
Every $1 \%$ of psyllium added resulted in an approximately $4 \%$ increase in the FWA value (Table 2 ). As expected, the peak time was higher $(8 \mathrm{~min})$ with WF than with WWF (6 min). Addition of higher amounts of bran or germ fractions lowered the peak time further. In contrast, addition of psyllium increased the peak time significantly (11 min). Dough stability values were higher for WF (16.5 min) than for WWF ( $9 \mathrm{~min})$. Fine bran addition to WF significantly decreased the dough's stability, whereas the addition of coarse bran had no effect on this parameter. Wheat germ addition drastically reduced the dough's stability ( $3.5 \mathrm{~min}$ ) because of the presence of reducing substances such as glutathione [9], which was also reflected in higher MTI values. Marti et al. [21] have investigated the weakening of dough 
containing wheat germ and have shown glutathione to be the major component responsible for the deterioration of rheological characteristics of dough. The reason for lower dough stability in WWF than in WF could be due to the activity of various proteolytic enzymes present in the aleurone layer which is a normal constituent of WWF. The same could be true when fine bran fraction is added to WF [9]. As dough stability and MTI values are inversely related, lower MTI values are desirable in Arabic bread making. Interestingly, the addition of psyllium at all levels of addition increased dough stability (11 min). In terms of dough stability, MTI, and valorimeter values, various combinations of WF, coarse bran , and psyllium produced dough characteristics which were quite desirable for Arabic bread making.

Psyllium fiber has recently been shown to influence the starch hydration properties, pasting behavior, and dough rheology even under cold conditions [22]. Highly fibrillated cellulose has a large surface area, and these smaller fibrils have significantly higher interaction with psyllium heteroxylan resulting in a compact structure [23]. Psyllium husk, a rich source of soluble fiber fraction made of heteroxylan, has been shown to possess remarkable water absorption capacity and gelling properties [24]. According to them, the main functional component (F60) of psyllium husk is a complex branched heteroxylan structure that possesses unique rheological properties and offers tremendous functional applications in food industry. Many of the effects on the psyllium addition to WF on the rheological properties can be explained due to these interactions between heteroxylans with the gluten proteins.

The addition of psyllium to WF at various levels (from 1 to $5 \%$ ) significantly increased the water absorption (FWA) and peak time but decreased the dough stability and valorimeter values (Table 2). The heteroxylans in psyllium husk have a tremendous amount of hydroxyl groups which have higher water binding capacity [24]. As expected, in the presence of $10 \%$ fine bran (FB), but with an increasing levels of psyllium husk addition, both the FWA and peak time increased significantly (Table 3 ). In the presence of fine bran and psyllium competing for available water in the system, the dough proteins obviously took longer time to develop viscoelastic structure. However, with higher level of $20 \%$ fine bran and increasing amounts of psyllium addition, both the FWA and peak time increased significantly (Table 4), but the dough stability increased significantly only at higher levels of psyllium (4 and 5\%). Similarly, the valorimeter values were significantly higher at 4 and $5 \%$ level of psyllium than the lower levels of 1 to $3 \%$ of psyllium $[25,26]$. Extractable phenolics and proteins obtained from wheat bran have been shown to give positive correlation with dough strength and dough development time by Navrotskyi et al. [27].

When coarse bran (CB) was added at 10\% level along with the increasing amounts of psyllium (from 1 to $5 \%$ ), both the FWA and peak time increased significantly (Table 5), but the dough stability decreased at all levels of psyllium addition with no change in valorimeter values. Interestingly, at higher levels of CB $(20 \%)$ in the presence of increasing levels of psyllium (1 to $5 \%$ ), the FWA, dough stability, peak time, and valorimeter values were significantly increased (Table 6).
The higher dough stability and valorimeter values at higher levels of psyllium and bran could be explained due to the interaction of ferulic acid present in wheat bran pentosans that strengthens the gluten proteins of wheat flour as suggested by various workers [28-30]. The arabinoxylan (AX) in wheat bran is also known to strengthen the AX gels which increase their ability to bind higher amounts of water during dough making [31-33]. Recently, Parenti et al. [12] have also shown the bran and germ to have a negative effect on the rheological performance of the dough.

3.3. Extensograph Characteristics. The extensibility (E), resistance to extension (R-to-E) and area (A) under the extensigram of dough were significantly higher in WF than the WWF (Table 7). Compared to control WF, the addition of fine bran both at 10 and $20 \%$ level significantly reduced the E, R-to-E, and A values for dough; however, the coarse bran addition at both the 10 and $20 \%$ level significantly decreased the extensibility, but increased the R-to-E and A values. The addition of wheat germ at both levels (because of its higher glutathione content) significantly increased $\mathrm{E}$ values and reduced R-to-E values but slightly increased the $A$ values when compared with the WF control sample (Table 7). Psyllium addition at varying levels from 1 to $5 \%$ to the WF decreased E values (from 25.9 to $14.1 \mathrm{~cm}$ ) but increased Rto-E values (from 11.9 to $17.1 \mathrm{~cm}$ ) and increased area (A) under the graph (from 213 to $229.6 \mathrm{~cm}^{2}$ ), thus indicating greater dough stability (Table 8 ). When psyllium husk was added along with the $10 \%$ levels of fine bran, significantly decreased the E values from 25.9 to $11.2 \mathrm{~cm}$, R-to-E values from 11.9 to $10.9 \mathrm{~cm}$, and A under the graph from 213 to $83 \mathrm{~cm}^{2}$, indicating a slight weakening of the dough (Table 9). Higher level of fine bran (20\%) addition along with psyllium husk further weakened the dough structure as shown by the decrease in $\mathrm{E}$ and $\mathrm{A}$ values, but no significant change in R-to-E values (Table 10). In case of addition of coarse bran (10 and 20\% levels) along with psyllium husk at all levels from 1 to $5 \%$, the influence on the extensibility, resistance to extension, and area under the curve decreased significantly (Tables 11 and 12). Coarse bran fractions originate from the outermost layers of wheat kernel during the roller flour milling process. These outer layers are known to contain small epicarp hairy structure on their surfaces which have a strong weakening influence on the gluten structure and can even lead to lower specific volume of the baked bread [34]. The increased amounts of arabinoxylans and fiber contents have been shown to decrease extensibility and increase the R-to-E. The higher amounts of bran fractions also increase the total protein content but decrease the levels of gluten concentrations $[35,36]$, and the bran addition also reduces the gluten polymerization, resulting in the breakdown of the gluten structure [34].

The addition of bran to flour has been shown to affect the rheological characteristics due to many reasons. The fiber constituents present in bran and psyllium compete with the available water during dough missing, with other major polymers, such as gluten and starch, thus hindering the strong gluten network, leading to lower resistance to extension, but weaker gluten with more extensibility [37-39]. Secondly, 
the ferulic acid present in water soluble pentosans has been shown to strengthen the gluten network [31, 32]. Most of the phenolic compounds are concentrated mainly in the outer layers of cereal grains which constitute bran [40]. The physical interaction of bran components with the gluten network is the major reason of the negative effect of fiber addition on the decreased extensibility values and higher R-to-E values [38]. Ahluwalia et al. [41] have studied the effect of psyllium addition on the rheological properties of wheat flour. With the addition of psyllium, they observed higher water absorption and stability but decreased mixing tolerance. Rao and Rao [42] have studied the effect of incorporating wheat bran on the rheological characteristics and breadmaking quality of flour. They used higher levels than our study and found that a maximum of $30 \%$ wheat bran could be added to flour to obtain an acceptable bread. At the higher level of dietary fiber (bran, psyllium) addition, gluten proteins of the WF can partially manage the negative effects of fiber addition on the dilution of gluten proteins $[35,36,39$, 43]. Similar trends in dough rheological characteristics were observed with various combinations of coarse bran and psyllium addition to the WF during this study or with chickpea flour addition in earlier studies $[44,45]$.

\section{Conclusions and Recommendations}

The results presented here in this paper bring out clearly the influence of adding coarse bran, fine bran, wheat germ, and psyllium husk on various rheological characteristics as measured by Farinograph and Extensograph. The major reasons for the weakening of the gluten structure are the competition for water between starch, gluten, and fiber constituents that is available in the dough system. This study has pointed out that the addition of psyllium husk, though reduced the extensibility, but did not affect the area under the curve adversely. The use of psyllium husk has, thus, overcome some of the negative effects of wheat germ and bran fractions on the rheological characteristics of the white flour dough. It was concluded that the use of psyllium husk will evidently help the bakers to produce nutritious and acceptable quality Arabic bread. The various phenolic compounds present in the bran layers need to be further investigated if they play any significant role in strengthening the gluten proteins during the dough mixing process. More research is also needed to suggest ways to eliminate or reduce the negative effects of wheat bran, wheat germ, and psyllium husk addition on dough rheology. Addition of these fiber sources is very important for the baking industry to produce bakery products rich in dietary fiber, vitamins, minerals, and antioxidant phytochemicals to provide health benefits to the consumers against many noncommunicable diseases, such as obesity, type 2 diabetes, cardiovascular diseases, and many types of cancers.

\section{Data Availability}

All the research data is included in the manuscript, and more data is available with the client, as it was a client sponsored project.

\section{Conflicts of Interest}

The authors declare that they have no known conflict of financial interests or personal relationships that could have appeared to influence the outcome of this research work.

\section{Acknowledgments}

The authors thank the Kuwait Foundation for the Advancement of Sciences (Grant no. FB023C) for funding this research project and the Kuwait Institute for Scientific Research for providing facilities for carrying out this research project. The authors also thank the Kuwait Flour Mills \& Bakeries Co. and its employees for all the help in supplying us with the wheat flour, bran, germ samples, and for sponsoring this project.

\section{References}

[1] L. Marquart, "Food focus 2002: hot topics in cereal nutrition," Cereal Foods World, vol. 47, no. 7, pp. 328-331, 2002.

[2] E. Decker, G. Beecher, J. Slavin, H. E. Miller, and L. Marquart, "Whole grains as a source of antioxidants," Cereal Foods World, vol. 47, no. 8, pp. 370-373, 2002.

[3] A. Adam, H. W. Lopez, M. Leuillet, C. Demigne, and C. Remesy, "Whole wheat flour exerts cholesterol-lowering in rats in its native form and after use in bread-making," Food Chemistry, vol. 80, pp. 337-344, 2003.

[4] J. L. Slavin, D. Jacobs, L. Marquart, and K. Wiemer, "The role of whole grains in disease prevention," Journal of American Dietetic Association, vol. 101, pp. 780-785, 2001.

[5] A. M. Pajari, S. Oikarinen, S. Grasten, and M. Mutanen, "Diets enriched with cereal brans or inulin modulate protein kinase $\mathrm{C}$ activity and isoenzyme expression in rat colonic mucosa," British Journal of Nutrition, vol. 84, pp. 635-643, 2000.

[6] F. Al-Awadi, Personal communication, Director, Nutrition Administration, Ministry of Health, Govt. of Kuwait, Kuwait, 1996.

[7] G. S. Ranhotra, J. A. Gelroth, K. Astroth, and E. S. Posner, "Distribution of total and soluble fiber in various millstreams of wheat," Journal of Food Science, vol. 55, no. 5, pp. 13491351, 1990.

[8] D. S. Grimes and C. Gordon, "Satiety value of whole meal and white bread," Lancet, vol. 2, p. 106, 1978.

[9] Y. Pomeranz, M. D. Shogren, and K. F. Finney, "White wheat bran and brewer's spent grains in high-fiber bread," Bakers' Digest, vol. 50, no. 6, pp. 35-38, 1976.

[10] AACC, Approved Methods of the AACC, American Association of Cereal Chemists, St. Paul, MN, USA, 10th edition, 2000.

[11] Y. Pomeranz, "Chemical composition of kernel structures," in Wheat: Chemistry and Technology, Y. Pomeranz, Ed., pp. 97158, AACC, St. Paul, Minnesota, USA, 1988.

[12] O. Parenti, L. Guerrini, and B. Zanoni, "Techniques and technologies for the breadmaking process with unrefined wheat flours," Trends in Food Science and Technology, vol. 99, pp. 152-166, 2020.

[13] A. Pauly, B. Pareyt, E. Fierens, and J. A. Delcour, "Wheat (Triticum aestivum L. and T. turgidum L. ssp. durum) kernel hardness: II. Implications for end-product quality and role of puroindolines therein," Comprehensive Reviews in Food Science \& Food Safety, vol. 12, no. 4, pp. 427-438, 2013. 
[14] Y. Hemery, M. Chaurand, U. Holopainen et al., "Potential of dry fractionation of wheat bran for the development of food ingredients, part I: influence of ultra-fine grinding," Journal of Cereal Science, vol. 53, no. 1, pp. 1-8, 2011.

[15] C. Liu, L. Liu, L. Li et al., "Effects of different milling processes on whole wheat flour quality and performance in steamed breadmaking," LWT-Food Science and Technology, vol. 62, no. $1,2015$.

[16] O. O. Oladunmoye, R. Akinoso, and A. A. Olapade, "Evaluation of some physical-chemical properties of wheat, cassava, maize, and cowpea flours for bread making," Journal of Food Quality, vol. 33, 708 pages, 2010.

[17] E. Li, S. Dhital, and J. Hasjim, "Effects of grain milling on starch structures and flour/starch properties," Starch/Staerke, vol. 66, pp. 15-27, 2014.

[18] O. Ozboy and H. Koksel, "Unexpected strengthening effects of a coarse wheat bran on dough rheological properties and baking quality," Journal of Cereal Science, vol. 25, pp. 77-82, 1997.

[19] M. L. Sudha, R. Vetrimani, and R. Leelavathi, "Influence of fibre from different cereals on the rheological characteristics of wheat flour dough and on biscuit quality," Food Chemistry, vol. 100, pp. 1365-1370, 2005.

[20] C. M. Rosell, J. A. Rojas, and C. B. de Barber, "Influence of hydrocolloids on dough rheology and bread quality," Food Hydrocolloids, vol. 15, no. 1, pp. 75-81, 2001.

[21] A. Marti, L. Torri, M. C. Casiraghi, L. Franzetti, and M. A. Pagani, "Wheat germ stabilization by heat-treatment or sourdough fermentation: effects on dough rheology and bread properties," LWT-Food Science and Technology, vol. 59, no. 2, pp. 1100-1106, 2014.

[22] M. Belorio, G. Marcondes, and M. Gomez, "Influence of psyllium versus xanthan gum in starch properties," Food Hydrocolloids, vol. 105, article 105843, 2020.

[23] Y. Ren, B. R. Linter, and T. M. Foster, "Cellulose fibrillation and interaction with psyllium seed husk heteroxylan," Food Hydrocolloids, vol. 104, article 105725, 2020.

[24] Y. Ren, G. R. Yakubov, B. R. Linter, W. MacNaughtan, and T. M. Foster, "Temperature fractionation, physicochemical and rheological analysis of psyllium seed husk heteroxylan," Food Hydrocolloids, vol. 104, article 105737, 2020.

[25] N. Liu, S. Ma, L. Li, and X. Wang, "Study on the effect of wheat bran dietary fiber on the rheological properties of dough," Grain \& Oil Science \& Technology, vol. 2, no. 1, pp. 1-5, 2019.

[26] A. L. Girard, T. Teferra, and J. M. Awika, "Effects of condensed vs hydrolysable tannins on gluten film strength and stability," Food Hydrocolloids, vol. 89, pp. 36-43, 2019.

[27] S. Navrotskyi, G. Guo, G. S. Baenziger, L. Xu, and D. J. Rose, "Impact of wheat bran physical properties and chemical composition on whole grain flour mixing and baking properties," Journal of Cereal Science, vol. 89, article 102790, 2019.

[28] T. Demuth, J. Betschart, and L. Nystrom, "Structural modifications to water-soluble wheat bran arabinoxylan through milling and extrusion," Carbohydrate Polymer, vol. 240, article 116328, 2020.

[29] A. Kaur, M. P. Yadav, B. Singh, S. Bhinder, and N. Singh, "Isolation and characterization of arabinoxylans from wheat bran and study of their contribution to wheat flour dough rheology," Carbohydrate Polymer, vol. 221, pp. 166-173, 2019.

[30] S. Zhan, Y. Ren, J. Liu, E. P. Fuerst, and H. Geng, "Genomewide association study of feruloyl arabinoxylan content in common wheat grain," Journal of Cereal Science, vol. 89, article 102787, 2019.

[31] J. S. Sidhu, P. Nordin, and R. C. Hoseney, "Reaction of 14Ccysteine with wheat flour water solubles under UV light," Cereal Chemistry, vol. 57, no. 6, pp. 380-382, 1980.

[32] D. Ercili Cura, R. Lantto, M. Lille, M. Andberg, K. Kruus, and J. Buchert, "Laccase-aided protein modification: effects on the structural properties of acidified sodium caseinate gels," International Dairy Journal, vol. 19, no. 12, pp. 737745, 2009.

[33] P. Wang, C. Hou, X. Zhao, M. Tian, and R. Yang, "Molecular characterization of water-extractable arabinoxylan from wheat bran and its effect on the heat-induced polymerization of gluten and steamed bread quality," Food Hydrocolloids, vol. 87, pp. 570-581, 2019.

[34] Z. Gan, T. Galliard, P. R. Ellis, R. E. Angold, and J. G. Vaughan, "Effect of the outer bran layers on the loaf volume of wheat bread," Journal of Cereal Science, vol. 15, no. 2, pp. 151-163, 1992.

[35] L. Garófalo, D. Vazquez, F. Ferreira, and S. Soule, "Wheat flour non-starch polysaccharides and their effect on dough rheological properties," Industrial Crops and Products, vol. 34, no. 2, pp. 1327-1331, 2011.

[36] A. Cappelli, E. Cini, L. Guerrini, P. Masella, G. Angeloni, and A. Parenti, "Predictive models of the rheological properties and optimal water content in doughs: an application to ancient grain flours with different degrees of refining," Journal of Cereal Science, vol. 83, pp. 229-235, 2018.

[37] Z. Gan, P. R. Ellis, J. G. Vaughan, and T. Galliard, "Some effects of non-endosperm components of wheat and of added gluten on whole meal bread microstructure," Journal of Cereal Science, vol. 10, no. 2, pp. 81-91, 1989.

[38] M. W. Noort, D. van Haaster, Y. Hemery, H. A. Schols, and R. J. Hamer, "The effect of particle size of wheat bran fractions on bread quality-evidence for fibre-protein interactions," Journal of Cereal Science, vol. 52, no. 1, pp. 59-64, 2010.

[39] H. Zhang, X. Zhang, X. R. Cao, M. Iftikhar, and J. Wang, "Semi-solid state fermentation and enzymatic hydrolysis impeded the destroy of wheat bran on gluten polymerization," LWT-Food Science and Technology, vol. 98, pp. 306313, 2018.

[40] J. S. Sidhu, Y. Kabir, and F. G. Huffman, "Functional foods from cereal grains," International Journal of Food Properties, vol. 10, no. 2, pp. 231-244, 2007.

[41] P. Ahluwalia, J. S. Sidhu, and A. Kaur, "Effect of isabgol addition as a source of dietary fiber on rheological properties of flour," Chemie Mikrobiologie Technologie und Lebensmittel, vol. 16, no. 1/2, pp. 34-38, 1994.

[42] P. Rao Haridas and H. M. Rao, "Effect of incorporating wheat bran on the rheological characteristics and bread making quality of flour," Journal of Food Science and Technology, vol. 28, no. 2, pp. 92-97, 1991.

[43] A. Cappelli, L. Guerrini, E. Cini, and A. Parenti, "Improving whole wheat dough tenacity and extensibility: a new kneading process," Journal of Cereal Science, vol. 90, article 102852, 2019. 
[44] T. A. Zafar, A. R. Allafi, D. Alkandari, and A. Al-Othman, "Rheological characteristics of wheat-chickpea composite flour doughs and effect of Amla powder (Phyllanthus emblica L.) addition on the functional properties of bread," Food Science and Technology International, pp. 1-12, 2020.

[45] T. A. Zafar, A. Aldughpassi, A. Al-Mussallam, and A. Al-Othman, "Microstructure of whole wheat versus white flour and wheat-chickpea flour blends and doughs: impact on the glycemic response of pan bread," International Journal of Food Science, vol. 2020, Article ID 8834960, 9 pages, 2020. 